\title{
RELEVANCE AND VERIFICATION
}

\author{
BEN BLUMSON
}

\begin{abstract}
A. J. Ayer's empiricist criterion of meaning was supposed to have sorted all statements into nonsense on the one hand, and tautologies or genuinely factual statements on the other. Unfortunately for Ayer, it follows from classical logic that his criterion is trivial - it classifies all statements as either tautologies or genuinely factual, but none as nonsense. However, in this paper I argue that Ayer's criterion of meaning can be defended from classical proofs of its triviality by the adoption of a relevant logic - an idea which is motivated because, according to Ayer, the genuinely factual statements are those which observation is relevant to.
\end{abstract}

\section{INTRODUCTION}

Logical positivism promised to solve all philosophical problems. According to the logical positivists, every meaningful statement was either analytic or verifiable by experience. Any other discourse either did not consist in statements, or was not meaningful at all - in other words, nonsense. With this taxonomy the logical positivists hoped to demarcate science and mathematics, on the one hand, from metaphysics and religion on the other. There

Date: October 20, 2020. 
was space for debate about where particular issues fit within the template. But the overall shape of philosophy was fixed.

To pursue their philosophical project, the logical positivists sought a criterion for distinguishing statements which were verifiable by experience from statements which were not. In particular, according to Alfred Jules Ayer's (1936, 39) first empiricist criterion of meaning:

... we may say that it is the mark of a genuine factual proposition ... that some experiential propositions can be deduced from it in conjunction with certain other premises without being deducible from those other premises alone.

'The absolute is perfect', for example, is not genuinely factual according to Ayer because, he thought, it has no observable consequences, even in combination with auxiliary assumptions.

Ayer's first criterion is designed to accommodate two kinds of genuinely factual statement. The first are observation statements, such as 'it's hot' or 'that's red', which are directly verifiable (Ayer calls these "experiential propositions" in the quote above). The rest are indirectly verifiable. This category is supposed to include simple generalisations such as 'all crows are black', negative existentials such as 'there is no alien life', but also laws of nature such as 'nothing moves faster than light' and theoretical posits, such as the existence of dark matter or fundamental particles. 
Ayer's first criterion was central to his positivist project. He quotes it directly, for example, in the following attack on theism $(1936,115)$ :

For if the existence of such a God were probable, then the proposition that he existed would be an empirical hypothesis. And in that case, it would be possible to deduce from it, and other empirical hypothesis, certain experiential propositions which were not deducible from those propositions alone. But in fact this is not possible.

His first criterion was also supposed to support his emotivist position in metaethics $(1936,106)$, as well as his views on the self $(1936,126)$, other minds $(1936,129)$, idealism $(1936,138)$, and his attack on metaphysics in general $(1936,41-5)$.

So it is unfortunate for Ayer that if deducibility is understood as entailment in classical logic, as he surely intended, his first criterion is trivial. Responding to a criticism by Isaiah Berlin $(1938,234)$ in the second edition of Language, Truth and Logic, Ayer (1946, 11-2) himself conceded that his first criterion:

... is far too liberal, since it allows meaning to any statement whatsoever. For given any statement $S$ and an observationstatement $O, O$ follows from $S$ and 'if $S$ then $O$ ' without following from 'if $S$ then $O$ ' alone. 
In response to this problem, Ayer $(1946,13)$ proposed a more complicated criterion, which retained the same general spirit as the first. However, in a famous review by Alonzo Church $(1949,53)$, Ayer's second criterion was also proven to be trivial, for much the same reasons as the first. Many have attempted to provide revisions of the criterion which escape triviality, but there is no consensus on whether any succeeds. ${ }^{1}$

However, I will argue in this paper that if deducibility in Ayer's first criterion is understood as entailment in relevant logic, then Ayer's criterion escapes triviality. Roughly, the idea behind relevant logic is that a conditional $A \rightarrow B$ is true only if $A$ is relevant to $B$. Formally, this idea manifests itself in the variable sharing principle, according to which $A \rightarrow B$ is a tautology only if some atomic sentence occurs in both $A$ and $B .^{2}$ Importantly

\footnotetext{
${ }^{1}$ See, for example, Hempel (1950, 49-50), O’Connor (1950), Brown and Watling (1951), Scheffler (1957, 384-8), Nidditch (1961), Scheffler (1964, 154n), Ullian (1965), Makinson (1965), Cornman (1967), Plantinga (1967, 164-7), Watanabe (1969, 534-5), Cohen (1975), Cohen (1980), Sanford (1981), Pokriefka (1983), Pokriefka (1984), Foster (1985, 14-21), Wright (1986), Lewis (1988a), Lewis (1988c), Wright (1993), Wright (1989), Suárez (1999), Suárez (2000), Yi (2001) and Yi (2003). Rudolf Carnap's (1936; 1950) criterion met a similar fate - see, for example, Hempel (1950, 51-3), Madden and Kiteley (1962, 72-7), Schlesinger (1964), Wójcicki (1966), Kaplan (1975), Creath (1976), Justus (2014), Lutz (2017), Bradley (2018) and Surovell (2019). For Moritz Schlick's (1936) similar criterion see, for example, Holdcroft (1983).

${ }^{2}$ See for example Anderson and Belnap (1976, 252); Mares (2004, 8); Priest (2008, 172).
} 
for the argument below, this involves rejecting some classical inference rules, as I explain in Section 2.

Relevant logic is relevant here because Ayer's criterion is essentially an elucidation of which statements observation is relevant to. As Ayer (1936, 39) himself puts it:

We say that the question that must be asked about any putative statement of fact is ... Would any observations be relevant to the determination of its truth or falsehood?

Of course, Ayer could not have had relevant logic in mind. Nevertheless, I will argue that the sense of relevance elucidated in relevant logic can capture the intuitive content of Ayer's criterion. ${ }^{3}$

\footnotetext{
${ }^{3}$ That Ayer's criterion involves an explication of relevance is also noted by Berlin, who complains "Relevance is not a precise logical category, and fantastic metaphysical systems may choose to claim that observation data are "relevant" to their truth. Such claims cannot be rebutted unless some precise meaning is assigned to the concept of relevance, which, because the word is used to convey an essentially vague idea, cannot be done" (1938, 233). Marcello Dascal $(1971,81)$ suggests Berlin's complaint could be answered by “...developing a logic of relevance that would provide the conceptual machinery needed for a precise and adequate formulation of the empiricist thesis...", but he develops this idea in a Gricean framework (Dascal, 1979). That Ayer's criteria involves an elucidation of relevance is also agreed by David Lewis (1988c), who takes Ayer's (second) criterion to be aimed at distinguishing statements which are "partly about" observation. However, Lewis (1988b) employs the same notion of partial aboutness to argue that relevant logic fails as an elucidation of relevance. In a similar vein, Gerhard Schurz $(1991,425)$ argues that an explication of relevance can be used to defend Carnap's criterion of empirical significance,
} 
This motivation commits me in this paper to relevant logic succeeding in elucidating relevance in at least one acceptable sense. However, I make no claim about whether relevant logic is superior to classical logic in general, or vice versa. Moreover, it is compatible with what I say here that it is appropriate to use classical entailment for some purposes and relevant logic for others (Beall and Restall, 2006, 49-59). But I make no claim about this position either. Likewise, I do not wish to defend logical positivism in general, or Ayer's criterion in particular, against any other objections, except the charge of triviality.

Section 2 briefly summarises the motivation for relevant logic. Sections 3-4 explain the classical proof of triviality, and why it is questionable from a relevant point of view. Sections 5-7 introduce a worlds semantics for relevant modal logics, for the purpose of presenting a countermodel to the classical proof in Section 8 - for illustration I use the relevant modal logic $N R$, the details of which are briefly summarised in Appendix A. Sections 9-11 respond to objections. Finally, Section 12 considers whether Ayer's criterion could be defended from triviality merely by adopting a paraconsistent, rather than a relevant, logic.

but also distinguishes his approach from that encapsulated in relevant logic $(1991,412-6)$. And although Neil Tennant is a proponent of relevant (and intuitionist) logic, his criterion of empirical significance is developed in a classical framework (Tennant, 2002, 355-402). 


\section{The Paradoxes of Implication}

Relevant logic is motivated by the desire to improve upon the material conditional. In classical logic, one can validly infer any conditional from its consequent. In other words, according to classical logic $B \vdash A \rightarrow B$ is a valid inference rule. However, this inference rule is intuitively invalid - for example, from 'I will die young', it is counterintuitive to infer 'even if I live healthily I will die young', since it may well be that by living healthily I am able to avoid my early death. For this reason, the inference rule $B \vdash A \rightarrow B$ is sometimes known as the positive paradox of material implication. ${ }^{4}$

Likewise, in classical logic one can validly infer any conditional from the negation of its antecedent. In other words, according to classical logic $\neg A \vdash$ $A \rightarrow B$ is a valid inference rule. But this inference rule is intuitively invalid too - for example, from ' $\mathrm{I}$ do not live healthily' it is counterintuitive to infer 'even if I live healthily I will die young', for the same reason as in the positive paradox. For this reason, the inference rule $\neg A \vdash A \rightarrow B$ is sometimes called the negative paradox of material implication.

The paradoxes of material implication can be avoided by a possible worlds semantics for conditionals. For example, one might replace the material conditional with a strict conditional, according to which $A \rightarrow B$ is true in a possible world $w$ if and only if at every possible world $v$ accessible from $w$ in which $A$ is true in $v, B$ is true in $v$. In this case, both the positive

\footnotetext{
${ }^{4}$ Notably, Ayer $(1946,17)$ writes of classical logic that “... given these rules the a priori proposition $q \rightarrow(p \rightarrow q)$ is necessarily true", suggesting he accepts the positive paradox.
} 
and negative paradoxes of material implication are avoided, since even if 'I live healthily' is false or 'I die young' is true in the actual world, there may well be accessible possible worlds in which 'I live healthily' is true but 'I die young' is false.

However, relevant logicians point out that adopting a strict conditional still leaves us with the paradoxes of strict implication. According to the possible world semantics for the strict conditional, any conditional with a tautologous consequent is itself a tautology, since its consequent is true in every possible world. Likewise, any conditional with a contradictory antecedent is a tautology - there is no possible world in which its antecedent is true and its consequent false, simply because there is no possible world in which its antecedent is true

In order to solve the paradoxes of strict implication, relevant logicians are also led to reject certain entailments. Most importantly for our purposes, in order to deny that $(A \wedge \neg A) \rightarrow B$, they are led to deny the validity of the corresponding inference rule $A, \neg A \vdash B$, known as explosion. But explosion follows from disjunction introduction, viz. $A \vdash A \vee B$, and disjunctive syllogism, viz. $A \vee B, \neg A \vdash B$, which leads to the rejection of disjunctive syllogism too. ${ }^{5}$ As we shall see in Sections $3-4$, it is three of these controversial inference rules - viz., the negative paradox, disjunctive syllogism

\footnotetext{
${ }^{5}$ See Lewis and Langford (1959, 250); Anderson and Belnap (1976, 163-7).
} 
and explosion - which underlie the classical proof of the triviality of Ayer's criterion.

\section{The Classical Proof}

In the passage quoted in the introduction, Ayer conceded that his first criterion allows meaning to any statement whatsoever, but - as David Lewis (1988a) has pointed out - his proof of this was incomplete, since it failed to consider the case in which a conditional does entail its own consequent. Moreover, this second case is especially important for our purposes since, as we shall argue below, it is in the second case that the proof can be resisted by adopting a relevant logic. For this reason, this section gives a detailed presentation of Lewis' completed version of the proof.

Lewis' proof proceeds via consideration of two cases. For the first case, suppose there is some observation statement $O$ such that the conditional $S \rightarrow O$ does not entail $O$ by itself. Then it follows from modus ponens -

viz., the inference rule $A, A \rightarrow B \vdash B-$ and Ayer's criterion that $S$ is a genuine factual proposition, since $O$ can be deduced from $S$ in conjunction with the conditional $S \rightarrow O$ by modus ponens, but cannot be deduced from $S \rightarrow O$ alone. So in the first case, it follows that $S$ is a genuine factual proposition. This is the case Ayer gave in the quotation, and - as I concede in Section 11 - the reasoning goes through even in many relevant logics.

However, for the second case, suppose instead that there is no observation statement $O$ such that $S \rightarrow O$ does not entail $O$, or in other words that every 
observation statement $O$ is entailed by $S \rightarrow O$. It follows from the negative paradox of material implication that every observation statement $O$ is such that $\neg S$ entails $S \rightarrow O$, and so it follows from the transitivity of entailment that every observation statement $O$ is such that $\neg S$ entails $O$ directly.

But some observation statements (for example, 'it's light' and 'it's dark') contradict each other. So if $\neg S$ entails every observation statement, then $\neg S$ entails a contradiction (for example, it entails 'it's light and it's dark'), and so $\neg S$ is itself a contradiction. But if $\neg S$ is a contradiction, then it follows that $S$ is a tautology. So in the second case, it follows that $S$ is a tautology or, in other words, analytic (Lewis, 1988a, 2).

So classically, it follows from the first criterion that every statement is either a genuine factual proposition or else a tautology. That means that according to classical logic, Ayer's supposed threefold distinction between nonsense, on the one hand, and tautologies and genuine factual statements on the other, collapses into a mere twofold distinction between tautologies and genuine factual statements. But this defeats the whole purpose of the criterion. In other words, classical logic entails that Ayer's first criterion is trivial - according to it, there is no nonsense.

\section{Variations of the Proof}

Since the negative paradox of material implication is highly counterintuitive, it would be natural to resist the classical proof of the triviality of Ayer's criterion by adopting, for example, a possible world semantics for 
conditionals. But adopting a different theory of conditionals would not suffice to resolve the underlying problem, since the proof of the triviality of the first criterion can simply be restated by replacing $S \rightarrow O$ in the proof with a different auxiliary statement in which no conditional occurs, in which case questions about the kind of conditional involved are irrelevant.

I will give two examples. For the first example consider again an arbitrary statement $S$. But for the first case suppose there is some observation statement $O$ such that the disjunction $\neg S \vee O$ does not entail $O$ by itself (instead of the conditional $S \rightarrow O$ as in the previous proof). Then it follows from Ayer's criterion that $S$ is a genuine factual statement, because $S$ and $\neg S \vee O$ entail $O$ by disjunctive syllogism, even though $\neg S \vee O$ does not entail $O$ on its own. So in the first case, $S$ is again a genuine factual statement.

For the second case suppose that every observation statement $O$ is such that $\neg S \vee O$ entails $O$. Then since every observation statement $O$ is such that $\neg S$ entails $\neg S \vee O$ by disjunction introduction - viz. the inference rule $A \vdash A \vee B$ - and since $\neg S \vee O$ entails $O$ (from the description of the case), it follows from the transitivity of entailment that $\neg S$ entails every observation statement $O$. But since some observation statements contradict each other, it follows for the same reason as before that $\neg S$ is a contradiction and so $S$ is a tautology. 
Of course, since in classical logic $S \rightarrow O$ and $\neg S \vee O$ are equivalent, this proof is classically equivalent to the proof in the previous section. Nevertheless, this variation shows that adopting a non-classical theory of conditionals which denies this equivalence would not suffice to avoid the problem for Ayer's criterion, since there is no conditional in $\neg S \vee O$, and so the second version of the proof would be left untouched by this manoeuvre. Rejecting the negative paradox of material implication is not enough to save Ayer's criterion from triviality.

For the second and final example consider again an arbitrary statement $S$. But for the first case suppose there is some observation statement $O$ such that the negation $\neg S$ does not entail $O$ by itself. Then it follows from Ayer's criterion that $S$ is a genuine factual statement, since $S$ and $\neg S$ entail $O$ by explosion, even though $\neg S$ does not entail $O$ by itself (Watanabe, 1969, 136-7). For the second case suppose that $\neg S$ does entail every observation statement $O$. Then $\neg S$ is a contradiction and $S$ a tautology for the same reason as before. In either case, $S$ is genuinely factual, or else a tautology.

As well as resisting the first variation of the proof by rejecting the negative paradox of material implication, relevant logics can reject both these variations by rejecting disjunctive syllogism and explosion. But of course, that does not suffice to show that Ayer's criterion is not trivial in relevant logic. For all we have said so far, and as the history of the problem strongly suggests, there may be some other auxiliary sentence, apart from $S \rightarrow O$, 
$\neg S \vee O$ or $\neg S$, which could be used to prove the criterion is trivial even by the lights of relevant logic.

\section{The Routley Star}

In order to show that Ayer's first criterion is not trivial according to relevant logic, we can construct a countermodel in which some observation sentences contradict each other, but not all sentences are tautologies or genuinely factual. To do this we need a semantics for relevant logic. For this purpose, we will adopt a possible worlds semantics not just for necessity and the conditional, but also for negation. This section introduces the ingredient in the semantics responsible for negation, known as the Routley star.

The Routley star is a binary operator $*$ which associates each world $w$ with a unique star-world, denoted by $w^{*}$. Whereas according to classical possible worlds semantics, $\neg A$ is true at $w$ if and only if $A$ is false at $w$, according to the star semantics, $\neg A$ is true at $w$ if and only if $A$ is false at $w^{*}$, the star-world of $w$. To ensure that $\neg \neg A$ and $A$ are equivalent, the star operator is constrained so that $w^{* *}=w$ for all worlds $w$ or, in other words, so that every world is its own star-world's star-world. ${ }^{6}$

The star semantics invalidates explosion, or in other words allows that $A$ and $\neg A$ do not entail $B$, since if $A$ is true and $B$ is false at a world $w$, but $A$ is false at world $w^{*}$, it will follow that $A$ and $\neg A$ are both true at $w$, even though $B$ is false at $w$. For the same reason, the star semantics invalidates

\footnotetext{
${ }^{6}$ See, for example, Routley and Routley $(1972,337-8)$.
} 
disjunctive syllogism, or in other words allows that $A$ and $\neg A \vee B$ do not entail $B$, since if $A$ is true and $B$ is false at world $w$ and $A$ is false at world $w^{*}$, then $A$ and $\neg A \vee B$ are both true at $w$ even though $B$ is false at $w$.

The logic consisting of the star semantics for negation, together with classical possible worlds semantics, but no conditional, is known as FDE or first degree entailment. ${ }^{7}$ Since both explosion and disjunctive syllogism are invalid in first degree entailment, it supports neither of the two variations from the previous section of the classical proof of the triviality of Ayer's criterion. Nevertheless, there are two problems for using first degree entailment to resist the proof of the triviality of Ayer's criterion.

Firstly, first degree entailment has no conditional. Moreover, if material implication $A \supset B$ were defined in first degree entailment as $\neg A \vee B$, as it is in classical logic, then modus ponens would simply be a disguised version of disjunctive syllogism, and the invalidity of disjunctive syllogism would entail the invalidity of modus ponens (Priest, 2008, 154). The invalidity of modus ponens would have the advantage of undermining the original version of the proof of triviality, but at unacceptable cost.

Secondly, there are no tautologies in first degree entailment, since it's possible to construct a countermodel to the truth of any sentence. For consider a world $w \neq w^{*}$ such that every atomic sentence is false at $w$ and also every atomic sentence is true at $w^{*}$. Then it follows by induction that

\footnotetext{
${ }^{7}$ See, for example, Routley and Routley (1972); Priest (2008, 142-62).
} 
every sentence is false at $w$ and true at $w^{*}$, since if $A$ is false at $w$ and true at $w^{*}$ then from the semantics for negation $\neg A$ is false at $w$ and true in $w^{*}$, and if $A$ and $B$ are false in $w$ and true in $w^{*}$ then $A \wedge B$ and $A \vee B$ are false in $w$ and true in $w^{*}$ too. So every sentence is false at $w$ (Routley and Routley, 1972, 338).

Recall that Ayer's criterion is supposed to classify all sentences into three categories - tautologies, genuinely factual statements and nonsense. The classical proof of triviality purported to show that according to Ayer's criterion, there are only two categories - there are tautologies and genuinely factual statements, but no nonsense. It doesn't help to defend the cogency of the criterion to recover the nonsense, if that comes at the cost of losing the tautologies. So first degree entailment is not appropriate for defending Ayer's criterion.

\section{The Ternary Relation}

In order to recover the validity of modus ponens, we can combine the star semantics for negation with the ternary semantics for the conditional, according to which $A \rightarrow B$ is true in $w$ if and only if for all worlds $u$ and $v$ such that Rwuv and $A$ is true in $u, B$ is true in $v$ (Routley and Meyer, 1973). The ternary semantics for relevant implication is a generalisation of the classical semantics for strict implication, since they are the same in the special case in which $u=v$ for all $w, u, v$ such that $R w u v$, but come apart in the general case in which $R w u v$ but $u \neq v$ for some $R w u v$. 
In addition to the relation $R$ and operator $*$, let there be a distinguished world 0 , interpreted as the actual world, and such that $u=v$ if and only if R0uv for all $u, v$ (Priest and Sylvan, 1992, 220). If we stipulate that an argument is valid just in case there is no interpretation at which the premises are true while the conclusion is false at 0 , we obtain the basic relevant logic known as $B$. As a special case, a sentence is a tautology of $B$ just in case there is no interpretation at which it is false at 0 , or in other words no interpretation at which it is false at the actual world.

Modus ponens is valid in $B$. For suppose $A$ and $A \rightarrow C$ are both true at world 0 . Then $R 000$ since $0=0$ and $R 0 u v$ for all $u, v$ such that $u=v$. So from the semantics for the conditional $C$ is true at 0 . Moreover, unlike in first degree entailment, there are tautologies in $B$. The law of identity - viz., $A \rightarrow A$ - is a tautology, for example, since for all $u, v$ if $R 0 u v$ then $u=v$ and so if $A$ is true at $u$ then $A$ is true at $v$. So this semantics for $B$ avoids both our previous problems for first degree entailment.

Stronger relevant logics can be obtained by adding further constraints to the ternary relation and star operator. ${ }^{8}$ But this version of the semantics for relevant logic is still not ideal for the defence of Ayer's criterion, for two reasons. The first reason is that the defence of Ayer's criterion demands a distinction between tautologies in virtue of meaning and tautologies in

\footnotetext{
${ }^{8}$ See, for example, Routley et al. (1982, 298-302); Priest (2008, 202-3).
} 
virtue of form. Truth at world 0 in every interpretation defines tautologies in virtue of form. But what of tautologies in virtue of meaning?

One possibility would be to define tautologies in virtue of meaning as sentences which are true at world 0 in the intended interpretation. But since we are treating 0 as the actual world, this would amount to collapsing the distinction between tautologies in virtue of meaning and mere contingent truths - exactly the kind of collapse we are trying to avoid. Alternatively, we could define tautologies in virtue of meaning as sentences which are true at all worlds in the intended interpretation.

But adopting this second definition would still amount to having no tautologies in virtue of meaning. Recall that part of the motivation behind relevant logic is to prevent a conditional from being true merely because its consequent is a tautology, and so avoid the positive paradox of strict implication. In order to achieve this using the ternary semantics, every tautology must be false in some possible world in the intended interpretation. But in that case there would be no tautologies in virtue of meaning under the second definition - again exactly the kind of collapse we are trying to avoid.

The second reason is that the defence of Ayer's criterion also demands a distinction between validity in virtue of meaning and validity in virtue of form. To see this, recall that in the original version of the proof of triviality, there were two cases. In the first case there was some observation statement $O$ such that $S \rightarrow O$ does not entail $O$. In this case, we agreed that the reasoning was impeccable even by the standards of relevant logic, and so $S$ 
is genuinely factually according to Ayer's criterion. So it was only in the second case that the original proof of triviality could be resisted.

But in the second case, $S \rightarrow O$ entails $O$ for every observation statement $O$. Should we think of this as entailment in virtue of meaning, or entailment in virtue of form? It cannot be entailment in virtue of form. Suppose, for example, that $S$ were an atomic sentence. Then $S \rightarrow O$ does not entail any observation statement $O$ in virtue of form, and we would be back in the first case. It would follow that every atomic sentence is genuinely factual according to Ayer's criterion. But once again, this is exactly the kind of collapse we are trying to avoid.

So the sense of entailment or deducibility relevant to Ayer's criterion has to be validity in virtue of meaning, rather than validity in virtue of form. But lack of an interpretation in which the premises are true but the conclusion is false at world 0 defines validity in virtue of form, rather than validity in virtue of meaning. In order to capture validity in virtue of meaning, we need an intended interpretation, so we can say that an argument is valid in virtue of meaning just in case in every world of the intended model in which the premises are true, so is the conclusion.

\section{Relevant Modal Logic}

In order to recover tautologies in virtue of meaning, we introduce a binary accessibility relation $M$ to govern necessity, such that $\square A$ is true at world $w$ just in case $A$ is true at all worlds $v$ such that $M w v$. Then we can define 
a tautology in virtue of meaning as a sentence which is true at all worlds accessible from the actual world or, in other words, a sentence true at all worlds $w$ such that $M 0 w$. A tautology in virtue of form can be defined as a sentence which is true at every world accessible from 0 in all interpretations. ${ }^{9}$

Even though every sentence is false at some world in the intended interpretation, there may still be tautologies in virtue of meaning according to this definition, since not every world in the intended interpretation will be accessible from the actual world. Likewise, given appropriate constraints on the star operator, the ternary relation, and the accessibility relation, there will also be tautologies in virtue of form, since some sentences will be true at every accessible world in every interpretation which meets the constraints.

Different constraints on the relations $M$ and $R$, together with their interactions with the $*$ operator, lead to different relevant modal logics, with different tautologies in virtue of form. For the sake of specificity, I've chosen the relevant logic $N R$, which is described in full in Appendix A. Because $N R$ is relatively strong, it has the advantage (for our purposes) of retaining many classical tautologies. But the argument in the next section will work

\footnotetext{
${ }^{9}$ Note that Routley and Meyer $(1972,55)$ define a tautology in virtue of form as a sentence which is true at world 0 in every interpretation. However, in the presence of the rule of necessitation, according to which if $\vdash A$ then $\vdash \square A$, any tautology in virtue of form in their sense is one in ours. And since $\square A \vdash A$ any tautology in virtue of form in our sense is one in theirs. So our definition is equivalent to theirs.
} 
for any relevant modal logics with different constraints too, as long as they satisfy Lemma 1 below.

An argument is valid in virtue of meaning just in case in every world (whether it is accessible from 0 or not) in the intended interpretation in which the premises are true, the conclusion is true. The argument from $S \rightarrow O$ to $O$ could be valid in virtue of meaning, even if $S$ is an atomic sentence, so long as $O$ is true in every world in the intended interpretation in which $S \rightarrow O$ is true. An argument is valid in virtue of form just in case in every interpretation in which the premises are true, the conclusion is true. ${ }^{10}$

In addition to $M$ and $R$ we need one further relation $\leq$, defined by $w \leq v=_{\text {def }}(\exists x)(M 0 x \wedge R x w v)$, which we interpret as being a relation of inclusion or parthood between worlds. This interpretation requires imposing the hereditariness constraint, according to which for all atomic sentences $p$ and worlds $w, v$ such that $w \leq v$, if $p$ is true at $w$ then $p$ is true at $v$. With other appropriate constraints, the hereditariness constraint entails:

Lemma 1 (Hereditariness). For all sentences $A$ and worlds $w, v$ such that $w \leq v$, if $A$ is true at $w$ then $A$ is true at $v$.

\footnotetext{
${ }^{10}$ For this definition of validity see Routley and Meyer $(1972,55)$ and Appendix A.
} 
In other words, if a world is part of another, everything true at the former is true in the latter. In the next section, we'll use the hereditariness lemma to prove that Ayer's first criterion is not trivial according to relevant logic. ${ }^{11}$

\section{A Countermodel}

In this section, I use the world semantics to give an interpretation in which there is a sentence $S$ which, according to Ayer's criterion, is neither a genuine factual statement nor a tautology. Let us suppose that $S$ is an atomic statement (say 'the absolute is perfect'), that $O$ is an atomic observation statement (say 'it's light'), and that $\neg O$ is also an observation statement (say 'it's not light' or, equivalently, 'it's dark'). For simplicity, let us also suppose that $O$ and $\neg O$ are the only observation statements.

Then consider an interpretation in which there are exactly six possible worlds: $0=0^{*}, i=i^{*}, j, k, j^{*}$ and $k^{*}$. In accordance with the constraints listed in Appendix A, let the accessibility relation $M$ be the transitive closure of the relation illustrated by the directed graph in Figure 1b and the ternary relation $R$ be as illustrated in Table 1 , where a letter $z$ appearing in row $x$ and column $y$ represents that Rxyz. Then the hereditariness relation is the transitive closure of the relation illustrated by the directed graph in Figure 1a or, in other words, is such that $j^{*}<0<j$ and that $k^{*}<i<k$ (where $w<v$ if and only if $w \leq v$ but not $v \leq w)$.

\footnotetext{
${ }^{11}$ For a proof of the hereditariness lemma for $N R$ see Routley and Meyer $(1972,56)$.
} 


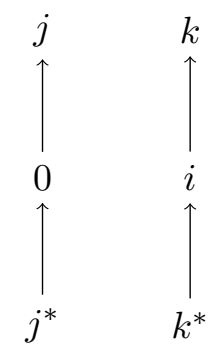

(A) Hereditariness

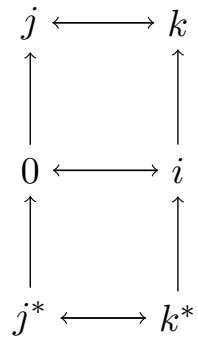

(B) Accessibility

FIGURE 1

\begin{tabular}{|c|c|c|c|c|c|c|}
\hline$R x y z$ & 0 & $i$ & $j$ & $k$ & $j^{*}$ & $k^{*}$ \\
\hline 0 & $0, j$ & & $j$ & & $0, j, j^{*}$ & \\
$i$ & & $i, k$ & & $k$ & & $i, k, k^{*}$ \\
$j$ & $j$ & & $j$ & & $0, j, j^{*}$ & \\
$k$ & & $k$ & & $k$ & & $i, k, k^{*}$ \\
$j^{*}$ & $0, j, j^{*}$ & & $0, j, j *$ & & $0, j, j^{*}$ & \\
$k^{*}$ & & $i, k, k^{*}$ & & $i, k, k^{*}$ & & $i, k, k^{*}$ \\
\hline
\end{tabular}

TABLE 1. The Ternary Relation

In accordance with the hereditariness constraint, let $S$ and $O$ be both false at $j^{*}$ and 0 , and $S$ be true but $O$ false at $j$, as illustrated in Table 2 . Likewise, let $S$ be false but $O$ true at both $k^{*}$ and $i$, and both $S$ and $O$ be true at $k$. It follows that $S$ and $\neg S$ are both false at $j^{*}$ and $k^{*}$ but both true at $j$ and $k$. The countermodel is complicated, but the underlying idea is simple - it is just to introduce worlds where $S$ and $\neg S$ are both true.

Note that in this interpretation, $S$ is not a tautology, since $S$ is false at 0 and $i$, even though 0 and $i$ are both accessible from 0 , or in other words both $M 00$ and $M 0 i$. To show that Ayer's criterion is not trivial in the relevant 


\begin{tabular}{|c|cccccc|}
\hline & $S$ & $O$ & $\neg S$ & $\neg O$ & $S \rightarrow O$ & $S \rightarrow \neg O$ \\
\hline 0 & & & $T$ & $T$ & & $T$ \\
$i$ & & $T$ & $T$ & & $T$ & \\
$j$ & $T$ & & $T$ & $T$ & & $T$ \\
$k$ & $T$ & $T$ & $T$ & & $T$ & \\
$j^{*}$ & & & & $T$ & & $T$ \\
$k^{*}$ & & $T$ & & & $T$ & \\
\hline
\end{tabular}

TABLE 2

$\operatorname{logic} N R$, it remains to be shown that in this interpretation, $S$ is not a genuine factual statement either. Since $S$ does not entail either observation statement on its own, the important question is then whether there is any other statement which entails either $O$ or $\neg O$ in combination with $S$, but does not entail either of them on its own.

Unlike in classical logic $\neg S$ will not do. Because $\neg S$ and $S$ are true at $j$, but $O$ is false at $j, \neg S$ and $S$ do not entail $O$. Likewise, because $\neg S$ and $S$ are true at $k$, but $\neg O$ is false at $k, \neg S$ and $S$ do not entail $\neg O$. The same reasoning shows that $\neg S \vee O$ and $S$ do not entail $O$ (and $\neg S \vee \neg O$ and $S$ do not entail $\neg O$ ). But what about the example in the original version of the classical proof of the triviality of Ayer's criterion, namely $S \rightarrow O$ (and $S \rightarrow \neg O) ?$

Since modus ponens is valid, $S \rightarrow O$ does entail $O$ in combination with $S$. But notice that there are exactly three worlds in which $O$ is false, viz.: $j^{*}, 0$ and $j$. And notice from Table 1 that $R j^{*} j j, R 0 j j$ and $R j j j$. Since $S$ is true and $O$ is false in $j$, it follows from the ternary semantics that $S \rightarrow O$ 
is false in all worlds in which $O$ is false. In other words, it follows that in all worlds in which $S \rightarrow O$ is true, $O$ is true, or that $S \rightarrow O$ entails $O$.

Likewise, although $S \rightarrow \neg O$ entails $\neg O$ in combination with $S$, parallel reasoning shows that $S \rightarrow \neg O$ entails $\neg O$ on it own. So neither $S \rightarrow O$ nor $S \rightarrow \neg O$ show that $S$ is a genuine factual statement, since although both do entail an observation statement in combination with $S$, both entail that observation statement on their own. To show that $S$ is a genuine factual statement, a different auxiliary statement is required.

But it is provable that there is no statement $X$ that in combination with $S$ entails $O$, without entailing $O$ on its own. For suppose there were. In order not to entail $O$ on its own, $X$ would have to be true in some world in which $O$ is false. So $X$ would have to be true at either $j^{*}, 0$ or $j$. But to entail $O$ in combination with $S, X$ would have to be false at every world in which $S$ is true but $O$ is false. So $X$ would have to be false in $j$. But this cannot be, because it follows from the hereditariness lemma that everything which is true at $j^{*}, 0$ or $j$ is true at $j$.

Likewise, there is no statement $X$ that in combination with $S$ entails $\neg O$, without entailing $\neg O$ on its own. For suppose there were. Then in order not to entail $\neg O$ on its own $X$ would have to be true in some world in which $\neg O$ is false. So $X$ would have to be true at either $k^{*}, i$ or $k$. But to entail $\neg O$ in combination with $S, X$ would have to be false at every world in which $S$ is true but $\neg O$ is false. So $X$ would have to be false in $k$. But this can't be, 
since the hereditariness lemma entails everything true at $k^{*}, i$ or $k$ is true at $k$.

Since $O$ and $\neg O$ are the only observation statements, it follows that there is no statement which in combination with $S$ entails an observation statement, but which does not entail that observation statement on its own. In

other words, $S$ is not a genuine factual statement according to Ayer's criterion. Nor, as we have seen, is $S$ a tautology. So Ayer's criterion, interpreted in terms of relevant entailment, does not entail that all statements are either genuinely factual or else tautologies.

\section{Objections to the Countermodel}

The countermodel in the previous section shows that in the relevant logic $N R$, Ayer's criterion does not entail that every statement is either genuinely factual or else a tautology. However, because the countermodel is a very simple one, it is not particularly realistic. This leads to several objections, which we address in this section. In each case, we argue that the unrealistic feature is merely an artefact of the model, which could be avoided by complicating the model in relatively straightforward ways.

Firstly, note that in this model $S \rightarrow O$ is true at world 0 . Since we are interpreting truth at world 0 as truth in the actual world, this means that according to the model $S \rightarrow O$ is true. However it's plausible that if $S$ is not genuinely factual and $O$ is an observation statement, then $S$ is irrelevant to $O$. And recall that relevant logics are supposed to capture the idea that if 
$S$ is irrelevant to $O$, then the conditional $S \rightarrow O$ is false. So according to a realistic model, $S \rightarrow O$ ought to be false, rather than true.

However, this unrealistic feature of the model could be avoided by introducing additional worlds $w, v$ into the model such that $S$ is true at $w, O$ is false at $v$ and $R 0 w v$. For similar reasons, although in the countermodel $S \rightarrow \neg O$ is true in world $i$, it's more plausible that $S \rightarrow \neg O$ should be false at world $i$. But this unrealistic feature of the model could be avoided by introducing additional worlds $w, v$ such that $S$ is true at $w, \neg O$ is false at $v$ and Riwv.

Secondly, note that in every world in the model in which $O$ is true $S \rightarrow O$ is also true, and so according to the countermodel $O$ entails $S \rightarrow O$ (as illustrated in Table 2). Likewise, in every world in the model in which $\neg O$ is true $S \rightarrow \neg O$ is also true, and so according to the countermodel $\neg O$ entails $S \rightarrow \neg O$. But these entailments are instances of the positive paradox of material implication, and the motivations canvassed in Section 2 for rejecting the validity of the positive paradox also extend to rejecting these entailments.

But the entailment from $O$ to $S \rightarrow O$ could be avoided by introducing an additional world $w$ into the model such that $O$ is true but $S \rightarrow O$ is false at $w$ (which would in turn require introducing worlds $u, v$ such that $S$ is true at $u, O$ is false at $v$ and $R w u v$ ). Likewise, the entailment from $\neg O$ to $S \rightarrow \neg O$ could be avoided by introducing an additional world $w$ into the model such that $\neg O$ is true but $S \rightarrow \neg O$ is false at $w$ (which would in turn 
require introducing worlds $u, v$ such that $S$ is true at $u, \neg O$ is false at $v$ and $R w u v)$.

Thirdly, note that $\neg S$ is true in the countermodel at every world accessible from 0 , viz. at $0, i, j$ and $k$ (as illustrated in Table $1 \mathrm{~b}$ and Table 2). Since we are interpreting being true at every world accessible from 0 as being a tautology, it follows that according to the countermodel $\neg S$ is a tautology, even though $S$ is genuinely factual. This feature could be avoided by altering the model so that $S$ is true and $\neg S$ is false at $i$. However, the constraints on $N R$ still entail that either $S$ or $\neg S$ is true in every world accessible from 0 , which is the basis of the first objection in the next section.

Fourthly, note that although there are worlds in the model where both $S$ and $\neg S$ are true, as well as worlds in the model where neither $S$ nor $\neg S$ are true, there are no worlds in the model where both $O$ and $\neg O$ are true nor where neither $O$ nor $\neg O$ are true. It follows that according to the model that $S \rightarrow O \vee \neg O$ and $O \wedge \neg O \rightarrow S$, for example, are tautologies. But these are instances of the paradoxes of strict implication, and the motivations canvassed in Section 2 for rejecting the validity of the positive paradox also extend to rejecting that they are tautologies.

But as for the other unrealistic features of the model, this could be avoided by introducing additional worlds. In particular, $S \rightarrow O \vee \neg O$ would not be a tautology if there were a world $w$ accessible from 0 and worlds $u, v$ such that $S$ is true at $u$, neither $O$ nor $\neg O$ is true at $v$, and Rwuv. Likewise, $O \wedge \neg O \rightarrow S$ would not be a tautology if there were a world $w$ accessible 
from 0 and worlds $u, v$ such that both $O$ and $\neg O$ is true at $u, S$ is false at $v$, and Rwuv. The same goes for any other fallacies of relevance which happen to be present in the model.

Finally, it's an unrealistic feature of the model that the only observation statements are $O$ and $\neg O$. This is important since Church's (1949) proof of the triviality of Ayer's second criterion, for example, relies not on there being at least two observation statements which contradict each other, but on there being at least three observation statements which are logically independent. Ensuring that these three observations statements are logically independent would require introducing even more worlds at which their truth-values differ, and so further complicate the countermodel.

\section{Objections to NR}

The objections in the previous section can all be addressed by complicating the countermodel. In this section, we turn to objections which would arise to any countermodel governed by the constraints of $N R$. In other words, in this section we address objections that arise due to our employment of $N R$, rather than some other relevant modal logic, for the purposes of illustration. In each case, we argue the objection could be avoided by adopting a relevant modal logic other than $N R$, which is governed by different constraints.

Firstly, if $S$ is not genuinely factual, then it's intuitive that neither $S$ nor its negation $\neg S$ should be true. Moreover, that statements which are 
not genuinely factual are neither true nor false has exegetical support in Langauge, Truth, and Logic. Ayer (1936, 31) writes, for example:

If a putative proposition fails to satisfy this principle [Ayer's first criterion], and is not a tautology, then I hold that it is metaphysical, and that, being metaphysical, it is neither true nor false, but literally senseless.

Given what Ayer says in this quotation, one would expect a logic which makes sense of his criterion would be one according to which sentences which are not genuinely factual are neither true nor false, or at least one in which neither they nor their negation are true.

On the face of it, $N R$ would seem to be such a logic, since its semantics allows for worlds in which both a sentence and its negation are false. According to the countermodel in Section 8, for example, neither $S$ nor $\neg S$ is true in worlds $j^{*}$ and $k^{*}$. However, this virtue is an illusion, since in order to ensure the law of excluded middle is a tautology - viz., that $\vdash A \vee \neg A-$ the constraints on interpretations of $N R$ are such that at every world accessible from 0 , either $A$ or $\neg A$ is true. According to the countermodel in Section 8, for example, neither $j^{*}$ nor $k^{*}$ are accessible from 0 .

Moreover, since 0 is accessible to itself, or in other words $M 00$, either $S$ or $\neg S$ must be true at 0 . And since we are interpreting 0 as the actual world, it follows that either $S$ or $\neg S$ must be in fact true - contrary to the idea that if $S$ is not genuinely factual, then neither $S$ nor $\neg S$ should be true. 
Likewise, since according to Ayer a statement is only true if it is a tautology or genuinely factual, if $S$ is not genuinely factual, then it follows that $\neg S$ must be true (as it is at world 0 in the countermodel).

This problem could be avoided by adopting a relevant modal logic weaker than $N R$, with less constraints on its models. But the cost of this would be losing the law of excluded middle as a tautology, and the law of excluded middle also has considerable exegetical support in Language, Truth and Logic. Ayer (1936, 79) writes, for example:

... the proposition 'either some ants are parasitic or none are' is analytic. For one need not resort to observation to discover that there either are or are not ants which are parasitic. If one knows what is the function of the words 'either', 'or', and 'not', then one can see that any proposition of the form 'either $p$ is true or $p$ is not true' is valid, independently of experience. Accordingly, all such propositions are analytic.

Given what Ayer say in this quotation, one would expect a logic which makes sense of his criterion to be one, like $N R$, which validates the law of excluded middle.

Whatever the case, what remains of this objection is an exegetical question about Ayer's system in Langauge, Truth and Logic, rather than a logical question about the cogency of his first criterion. In the family of relevant logics characterised by varying the constraints in the semantics above, there 
are some which allow both a statement and its negation to be false, and others which validate the law of excluded middle. Outside that family, there are logics - such as, for example, supervaluationism - which both validate the law of excluded middle and allow both a statement and its negation to be neither true nor false. I leave it to the reader to decide which logic, if any, would make the best sense of Ayer's intentions.

The second objection is that if $S$ is not genuinely factual, there must be worlds in the model not only where $S$ is true, but where $S$ and $\neg S$ are both true. This feature of the countermodel is inescapable. If there were no worlds where both $S$ and $\neg S$ are true, and there is some observation statement $O$ not entailed by $S$ alone, then it is vacuously true that in every world in which $S$ and $\neg S$ are both true, $O$ is true, and so $S$ and $\neg S$ vacuously entail $O$. Then according to Ayer's criterion, it would follow that as long as $S$ does not entail all observation statements, $S$ is genuinely factual.

However, this is not so much an objection, as a restatement of the basic idea used by the worlds semantics for relevant logics to invalidate explosion. In other words, the fact that in order to prevent a piece of nonsense $S$ and its negation $\neg S$ from entailing an observation statement $O$, there must be a world where $S$ and $\neg S$ are both true but $O$ is false, is simply a manifestation of the more general idea that in order to prevent a statement $A$ and its negation $\neg A$ from entailing some other statement $B$, there must be a world in which $A$ and $\neg A$ are both true but $B$ is false. 
But in Section 8, this issue was exacerbated since $i$ and $j$, the two worlds in the countermodel in which $S$ and $\neg S$ are both true, are accessible from 0 . Since we are interpreting accessibility from world 0 as possibility, it follows that according to the countermodel, it is possible that $S$ and $\neg S$ are both true. But it might be argued that although there must be worlds where $S$ and $\neg S$ are both true, these should not be possible worlds, but at most impossible worlds. The only possible worlds in the countermodel, according to this objection, should be 0 and $i$, the classical ones.

One could address this issue by varying the accessibility relation $M$ in the countermodel, so that worlds $j$ and $k$ are no longer accessible from 0 . This would come at the cost of violating some of the constraints imposed on models of $N R$, and so would amount to the adoption of a different logic. ${ }^{12}$ But so long as the constraints were still sufficient to entail the hereditariness lemma, and so long as the hereditariness relation $\leq$ was still as as in Section 8, the argument in Section 8 supporting the non-triviality of Ayer's first criterion would still go through.

\section{General Objections}

In this section, we turn to more general objections, which would arise no matter how the countermodel were constructed, or which constraints we adopted. For the first, recall from section Section 3 that if there is some observation statement such that $S \rightarrow O$ does not entail $O$, then it follows

\footnotetext{
${ }^{12}$ See, for example, Mares $(2004,100-8)$ for discussion of the accessibility relation.
} 
from modus ponens and Ayer's criterion that $S$ is genuinely factual. So in any logic in which modus ponens is valid, it follows from the reasoning in Section 3 that if a statement $S$ is not genuinely factual, then for every observation statement $O$, the conditional $S \rightarrow O$ entails $O$.

Is this consequence defensible? I argue that it is. Recall that in classical logic, if $A$ is a tautology, then for every statement $C$, the conditional $A \rightarrow C$ entails $C$. One might argue that this is acceptable since, because a tautology "says nothing", the whole force of an assertion with a tautology as its consequent is simply that the consequent is true - one might argue that 'if $1+1=2$, then I will have raisin toast for breakfast tomorrow', for example, is (classically) simply a long winded way of saying that I will have raisin toast for breakfast tomorrow.

Likewise, if a statement is not genuinely factual, then it "says nothing" about observation, and so the whole force of asserting a conditional with that statement as its antecedent and an observation statement as its consequent is to assert the observation statement itself. To say 'if the Absolute is sick, then it's dark', according to this line of thought, is (since 'the Absolute is sick' does nothing to qualify the observation in the consequent) simply a long winded way of saying that it's dark.

One might seek to buttress this defence by the observation that according to the countermodel in Section $8, S \rightarrow O$ is equivalent to $O$, since $S \rightarrow O$ and $O$ are true in all of the same worlds of the countermodel (as illustrated in Table 2). However, although we are committed to the entailment from 
$S \rightarrow O$ to $O$, recall that in Section 9 it was conceded that the entailment from $O$ to $S \rightarrow O$ is an unrealistic feature of the model, so only one direction of the entailment holds. So realistically, $S \rightarrow O$ is stronger than, rather than equivalent to, $O$.

The second objection, pointed out by Carl Hempel $(1950,50)$, is that even if $N$ is nonsense but $G$ is genuinely factual according to Ayer's criterion, the conjunction $N \wedge G$ will still be genuinely factual, since whatever observation statement is entailed by $G$ in conjunction with any auxiliary hypotheses, is also entailed by $N \wedge G$ together with those same auxiliary hypotheses. But it's intuitive that if $N$ is nonsense then $N \wedge G$ is nonsense too - if 'the absolute is perfect' is nonsense then so, according to Hempel $(1950,48)$ is 'all swans are white and the absolute is perfect'. Is this consequence defensible?

I argue that it is. Recall that according to Ayer, the genuinely factual statements are those that observation is relevant to. But if observation is relevant to 'all swans are white', then observation is relevant to 'all swans are white and the absolute is perfect' in virtue of being relevant to its first conjunct, even if it is not relevant to 'the absolute is perfect'. In general, observation is relevant to conjunctions of nonsense and genuinely factual statements, in virtue of being relevant to their genuinely factual conjuncts.

The third objection is that if $N$ is nonsense but $G$ is genuinely factual and true, then the disjunction $N \vee G$ will not be genuinely factual, but will be true. $N \vee G$ will not be genuinely factual, since whatever observation statement is entailed by $N \vee G$ in conjunction with auxiliary hypotheses, 
is also entailed by $N$ in conjunction with those same auxiliary hypothesis, so $N \vee G$ is genuinely factual only if $N$ is - but, by hypothesis, $N$ is not genuinely factual. But $N \vee G$ is true, simply because $G$ is true. This contradicts Ayer's claim that only genuinely factual statements are true.

Whereas Ayer could respond to the previous objection by granting that $N \wedge G$ is genuinely factual, he cannot grant that $N \vee G$ is not genuinely factual, unless he is willing to give up his claim that only tautologies or genuinely factual statements are true. Moreover, the reasoning just offered in support of the claim that $N \wedge G$ is genuinely factual also supports the claim that $N \vee G$ is genuinely factual - observation is relevant to disjunctions of nonsense and genuinely factual statements, in virtue of being relevant to their genuinely factual conjuncts.

How should Ayer respond to this objection? He could grant that $N \vee G$ is not genuinely factual and also true, thus abandoning his logical positivism. He could alter his criterion, or alter the background logic even further, so that $N \vee G$ is genuinely factual after all. Or he could alter the background logic so that although $N \vee G$ is not genuinely factual, it is not true either, even when $G$ is. But either change to the background logic would require more than adjusting the constraints on the star operator or the ternary and accessibility relations, and so take us beyond the family of relevant logics considered here. 


\section{Paraconsistent Logic}

So far, we have shown that adopting a relevant logic such as $N R$ is sufficient to avoid the classical proof of the triviality of Ayer's first criterion. But is it necessary? As we saw in Section 4, it is necessary to reject the inference rule of explosion. Logics which reject explosion - including relevant logics such as $N R$ - are known as paraconsistent logics. But not all paraconsistent logics are relevant logics. So one might wonder whether it suffices to defend Ayer's criterion from triviality to adopt a logic which is merely paraconsistent, rather than relevant.

This would be helpful in part because some paraconsistent logics have a three-valued semantics much simpler than the star-ternary semantics for $N R$. The paraconsistent logic $L P$ (for "logic of paradox"), for example, has a simple semantics with three truth-values: true, false and both. Then both negation and disjunction can be given a simple truth-functional semantics, as illustrated in Table 3 (Priest, 1979, 226-7). According to Subtable 3a, for example, the negation of a true sentence is false and the negation of a false sentence is true - just as in classical logic - but the negation of a sentence which is both is also both.

An argument is valid in $L P$ just in case the premises cannot all be either true or both while the conclusion is false; a sentence is a tautology just in case it must be true or both (Priest, 1979, 228). It follows that explosion is invalid in $L P$, or in other words $A, \neg A \nvdash B$, since if $A$ is both, then $\neg A$ is 


\begin{tabular}{|c|c|}
\hline & $\neg$ \\
\hline true & false \\
both & both \\
false & true \\
\hline
\end{tabular}

(A) Negation

\begin{tabular}{|c|ccc|}
\hline$\vee$ & true & both & false \\
\hline true & true & true & true \\
both & true & both & both \\
false & true & both & false \\
\hline
\end{tabular}

(B) Disjunction

TABle 3. Truth-Tables for $L P$

both even while $B$ is false. Likewise, disjunctive syllogism is invalid, or in other words $A, \neg A \vee B \nvdash B$ since when $A$ is both, $\neg A \vee B$ is also both (since its first disjunct $\neg A$ is both), even while $B$ is false.

Like first degree entailment, $L P$ has no conditional. And like first degree entailment, if material implication $A \supset B$ were defined in $L P$ as $\neg A \vee B$, then modus ponens would simply be a disguised version of disjunctive syllogism, and the invalidity of the latter would entail the invalidity of the former (Priest, 1979, 232-3). So as for first degree entailment, the invalidity of modus ponens would have the advantage of undermining the original version of the proof of triviality, but at unacceptable cost. (Unlike, first degree entailment, $L P$ at least has the advantage of possessing tautologies.)

In order to recover the validity of modus ponens, we can add to $L P$ a different truth-functional conditional. The logic $R M_{3}$ (for "three-valued relevant mingle"), for example, is obtained by adding to $L P$ the three-valued truth-function illustrated in Table $4 .^{13}$ The $R M_{3}$ conditional is false when

\footnotetext{
${ }^{13}$ See, for example, Anderson and Belnap $(1976,470)$ and Priest $(2008,125)$
} 


\begin{tabular}{|c|ccc|}
\hline$\rightarrow$ & true & both & false \\
\hline true & true & false & false \\
both & true & both & false \\
false & true & true & true \\
\hline
\end{tabular}

TABle 4. $R M_{3}$ Conditional

its antecedent is both and its consequent is false in order to validate modus ponens (and false when its antecedent is true and its consequent is both in order to validate modus tollens, viz. the inference rule $A \rightarrow B, \neg B \vdash \neg A$ ).

Notice that $R M_{3}$ invalidates the negative paradox of material implication, since when $A$ is both and $B$ is false, $\neg A$ is both even while $A \rightarrow B$ is false. Moreover, $R M_{3}$ invalidates the positive paradox of material implication, since when $A$ is true but $B$ is both, then $B$ is both even while $A \rightarrow B$ is false. And it invalidates both paradoxes of strict implication. Nevertheless, $R M_{3}$ is not quite a relevant logic, since it still validates the irrelevant $(A \wedge \neg A) \rightarrow$ $(B \vee \neg B)$, thus violating the variable sharing constraint.

But despite invalidating the negative paradox, a simple variation of the classical proof shows that Ayer's first criterion is trivial even according to $R M_{3}$. For the first case, suppose there is some observation statement $O$ such that the conditional $S \rightarrow O$ does not entail by itself. Then it follows from modus ponens and Ayer's criterion that $S$ is genuinely factual, just as in the classical case. So for the second case, suppose that for all observation statements $O$, the conditional $S \rightarrow O$ entails $O$. 
Notice from the bottom row of the truth-table that if $S$ is false, then $S \rightarrow O$ is true. Since $S \rightarrow O$ entails $O$ for all observation statements $O$, it follows that if $S$ is false, then all observation statements $O$ are true or both. But it is impossible that all observation statements are true or both. So it is impossible that $S$ is false. So it is necessary that $S$ is true or both. In other words, $S$ is a tautology. So according to $R M_{3}$, for all sentences $S$, either $S$ is genuinely factual or a tautology.

Notice that this variation of the proof relies on the claim that it is impossible that all observations statements are true or both. But by the lights of $R M_{3}$ this claim is disputable - since $R M_{3}$ allows for true contradictions, a proponent of $R M_{3}$ could argue that it is possible for all observation statements to be true or both. However, it is indisputable that not all observation statements are in fact true or both. From this it follows that $S$ is not in fact false. In other words, it follows that $S$ is in fact true or both. So from this weakened premise it still follows that for all sentences $S$, either $S$ is genuinely factual or else $S$ is true.

It follows from the example of $R M_{3}$ that although it's necessary to adopt a paraconsistent logic in order to avoid the triviality of Ayer's first criterion, it is not sufficient. Of course, it doesn't follow that it is necessary to adopt a relevant logic - for all I have said, there may be some other paraconsistent but not relevant logic which does avoid the triviality of Ayer's criterion. Likewise, for all I have said, there may be relevant logics other than $N R$ according to which Ayer's criterion is trivial after all. 
Nevertheless, the combined success of $N R$ and failure of $R M_{3}$ suggests that the motivation we offered in the introduction - according to which relevant logics are relevant here in part because Ayer's first criterion is intended as an explication of which statements observation is relevant to - is on the right track. The occurrence of paradigm examples of relevantly invalid inference rules in the classical proof of the triviality of Ayer's criterion, according to this line of thought, is not merely a coincidence, but symptomatic of an underlying cause. ${ }^{14}$

\section{Appendix A. $N R$}

This appendix states the worlds semantics semantics for $N R$ formally.

Definition 1. (Routley and Meyer, 1972, 54) An NR-structure is a quintuple $\langle W, 0, M, R, *\rangle$, where $W$ is a set of worlds, 0 is a world in $W, M$ is a binary relation on $W, R$ is a ternary relation on $W, *$ is a binary operator on $W$, and such that for all $u, v, w, t$, where $w \leq v={ }_{\operatorname{def}}(\exists x)(M 0 x \wedge R x w v)$ :

(1) $M w w$

(2) $(M u v \wedge M v w) \rightarrow M u w$
M-reflexivity $M$-transitivity

\footnotetext{
${ }^{14}$ This paper was presented in the Logic Seminar Series at the National University of Singapore in 2018 and at the Australasian Association of Logic conference at the University of Wollongong in 2019. I'm especially grateful to Zach Barnett, Bob Beddor, Theresa Helke, Frank Jackson, Kee Wei Loo, Qu Hsueh Ming, Michael Pelczar, Lavinia Picollo, Abelard Podgorski, Dilip Raghavan, Mary Salvaggio, Alexander Sandgren, Neil Sinhababu, Shawn Standefer, Weng Hong Tang, Chong Chi Tat, Joshua Thong, Daniel Waxman and Yang Yue for discussion.
} 
(3) Rwww

(4) $(\exists x)(R w u x \wedge R x v t) \rightarrow(\exists y)(R w v y \wedge R y u t)$

(5) $(\exists x)(R w u x \wedge M x v) \rightarrow(\exists x)(\exists y)(M w x \wedge M u y \wedge R x y v)$

(6) $w \leq w$

(7) $(w \leq v \wedge M v u) \rightarrow M w u$

(8) $(w \leq v \wedge R v u t) \rightarrow R w u t$

(9) $a^{* *}=a$

(10) $R w u v \leftrightarrow R w v^{*} u^{*}$.
$R$-reflexivity

Pasch's law

$$
\begin{array}{r}
\leq \text {-reflexivity } \\
M \text {-montonicity } \\
R \text {-monotonicity } \\
\text { *-involution }
\end{array}
$$

Definition 2. (Routley and Meyer, 1972, 54-5) An NR-interpretation on an $N R$-structure $\langle W, 0, M, R, *\rangle$ is a function $v$ from atomic sentences and worlds to truth-values $\{1,0\}$ such that for all worlds $w, u \in W$, atomic sentences $p$, and sentences $A, B$ :

(1) if $w \leq u$ and $v_{w}(p)=1$ then $v_{u}(p)=1 \quad$ hereditariness constraint

(2) $v_{w}(\neg A)=1$ if and only if $v_{w^{*}}(A)=0$

(3) $v_{w}(A \wedge B)=1$ if and only if $v_{w}(A)=1$ and $v_{w}(B)=1$

(4) $v_{w}(A \vee B)=1$ if and only if $v_{w}(A)=1$ or $v_{w}(B)=1$

(5) $v_{w}(A \rightarrow B)=1$ iff for all $x, y$ s.t. $R w x y v_{x}(A)=0$ or $v_{y}(B)=1$

(6) $v_{w}(\square A)=1$ if and only if all $x$ such that $M w x, v_{x}(A)=1$.

Definition 3. (Routley and Meyer, 1972, 55) $A$ entails $B$ according to interpretation $v$ if and only if for all $w \in W$ if $v_{w}(A)=1$ then $v_{w}(B)=1$. $A$ entails $B$ according to $N R$ if and only if $A$ entails $B$ according to all $N R$-interpretations $v$. 


\section{REFERENCES}

Anderson, A. and Belnap, N. (1976). Entailment, Vol. 1: The Logic of Relevance and Necessity, Princeton University Press, Princeton.

Ayer, A. J. (1936). Language, Truth and Logic, London: Victor Gollancz.

Ayer, A. J. (1946). Language, Truth and Logic, second edn, London: Victor Gollancz.

Beall, J. C. and Restall, G. (2006). Logical Pluralism, Oxford University Press, Oxford.

Berlin, I. (1938). Verification, Proceedings of the Aristotelian Society 39: $225-248$.

Bradley, D. (2018). Carnap's Epistemological Critique of Metaphysics, Synthese 195(5): 2247-2265.

Brown, R. and Watling, J. (1951). Amending the Verification Principle, Analysis 11(4): 87-89.

Carnap, R. (1936). Testability and Meaning, Philosophy of Science 3(4): 419-471.

Carnap, R. (1950). Empiricism, Semantics, and Ontology, Revue Internationale de Philosophie 4(11): 20-40.

Church, A. (1949). Review of Language, Truth and Logic, The Journal of Symbolic Logic 14(1): 52-53.

Cohen, L. J. (1975). Why Should the Science of Nature be Empirical?, Royal Institute of Philosophy Lectures 9: 168-183. 
Cohen, L. J. (1980). Is a Criterion of Verifiability Possible?, Midwest Studies In Philosophy 5(1): 347-352.

Cornman, J. W. (1967). Indirectly Verifiable: Everything or Nothing, Philosophical Studies 18(4): 49-56.

Creath, R. (1976). On Kaplan on Carnap on Significance, Philosophical Studies 30(6): 393-400.

Dascal, M. (1971). Empirical Significance and Relevance, Philosophia 1(1): 81-106.

Dascal, M. (1979). Conversational Relevance, in A. Margalit (ed.), Meaning and Use: Papers Presented at the Second Jerusalem Philosophical Encounter April 1976, Synthese Language Library, Springer, Dordrecht, pp. $153-174$.

Foster, F. (1985). Ayer, second edn, Routledge, London.

Hempel, C. G. (1950). Problems and Changes in the Empiricist Criterion of Meaning, Revue Internationale de Philosophie 4(11): 41-63.

Holdcroft, D. (1983). Schlick and the Verification Theory of Meaning, Revue Internationale de Philosophie 37(144/145 (1/2)): 47-68.

Justus, J. (2014). Carnap's Forgotten Criterion of Empirical Significance, Mind 123(490): 415-436.

Kaplan, D. (1975). Significance and Analyticity: A Comment on Some Recent Proposals of Carnap, in J. Hintikka (ed.), Rudolf Carnap, Logical Empiricist, Springer Netherlands, Dordrecht, pp. 87-94. 
Lewis, C. I. and Langford, C. H. (1959). Symbolic Logic, second edn, Dover, New York.

Lewis, D. (1988a). Ayer's First Empiricist Criterion of Meaning: Why Does It Fail?, Analysis 48(1): 1-3.

Lewis, D. (1988b). Relevant Implication, Theoria 54(3): 161-174.

Lewis, D. (1988c). Statements Partly About Observation, Philosophical Papers 17(1): 1-31.

Lutz, S. (2017). Carnap on Empirical Significance, Synthese 194(1): 217252.

Madden, E. H. and Kiteley, M. J. (1962). Postulates and Meaning, Philosophy of Science 29(1): 66-78.

Makinson, D. (1965). Nidditch's Definition of Verifiability, Mind LXXIV(294): 240-247.

Mares, E. (2004). Relevant Logic: A Philosophical Interpretation, Cambridge University Press, Cambridge.

Nidditch, P. (1961). A Defence of Ayer's Verifiability Principle Against Church's Criticism, Mind 70(277): 88-89.

O'Connor, D. J. (1950). Some Consequences of Professor A. J. Ayer's Verification Principle, Analysis 10(3): 67.

Plantinga, A. (1967). God and Other Minds: A Study of the Rational Justification of Belief in God, Cornell University Press, Ithaca.

Pokriefka, M. L. (1983). Ayer's Definition of Empirical Significance Revisited, Analysis 43(4): 166-170. 
Pokriefka, M. L. (1984). More on Empirical Significance, Analysis 44(2): 9293.

Priest, G. (1979). The Logic of Paradox, Journal of Philosophical Logic 8(1): $219-241$.

Priest, G. (2008). From If to Is: An Introduction to Non-Classical Logic, Cambridge University Press, Cambridge.

Priest, G. and Sylvan, R. (1992). Simplified Semantics for Basic Relevant Logics, Journal of Philosophical Logic 21(2): 217-232.

Routley, R. and Meyer, R. (1972). The Semantics of Entailment II, Journal of Philosophical Logic 1(1): 53-73.

Routley, R. and Meyer, R. (1973). The Semantics of Entailment, in H. Leblanc (ed.), Studies in Logic and the Foundations of Mathematics, Vol. 68 of Truth, Syntax and Modality, North-Holland, Amsterdam, pp. 199-243.

Routley, R., Plumwood, V., Meyer, R. K. and Brady, R. T. (1982). Relevant Logics and Their Rivals, Ridgeview, Atascadero.

Routley, R. and Routley, V. (1972). The Semantics of First Degree Entailment, Nous 6(4): 335-359.

Sanford, D. H. (1981). Superfluous Information, Epistemic Conditions of Inference, and Begging the Question, Metaphilosophy 12(2): 145-158.

Scheffler, I. (1957). Prospects of a Modest Empiricism, I, The Review of Metaphysics 10(3): 383-400. 
Scheffler, I. (1964). The Anatomy of Inquiry: Philosophical Studies in the Theory of Science, Routledge, London.

Schlesinger, G. (1964). The Formalization of Empirical Significance, Philosophy of Science 31(1): 65-67.

Schlick, M. (1936). Meaning and Verification, The Philosophical Review 45(4): 339-369.

Schurz, G. (1991). Relevant Deduction: From Solving Paradoxes Towards a General Theory, Erkenntnis 35(1-3): 391-437.

Suárez, A. G. (1999). Nuevos Problemas Y Cambios En El Criterio Empirista De Significación, Teorema: Revista Internacional de Filosofía 18(2): 19-31.

Suárez, A. G. (2000). The Verification Principle: Another Puncture, Analysis 60(3): 293-295.

Surovell, J. (2019). Empirical Significance, Predictive Power, and Explication, Synthese 196(6): 2519-2539.

Tennant, N. (2002). The Taming of the True, Oxford University Press, Oxford.

Ullian, J. (1965). A Note on Scheffler on Nidditch, The Journal of Philosophy 62(10): 274-275.

Watanabe, S. (1969). Knowing and Guessing, Wiley, New York.

Wójcicki, R. (1966). Semantical Criteria of Empirical Meaningfulness, Studia Logica: An International Journal for Symbolic Logic 19: 75-109. 
Wright, C. (1986). Scientific Realism, Observation and the Verification Principle, Fact, Science and Morality, Blackwell, Oxford, pp. 247-274.

Wright, C. (1989). The Verification Principle: Another Puncture-Another Patch, Mind 98(392): 611-622.

Wright, C. (1993). Scientific Realism and Observation Statements, International Journal of Philosophical Studies 1(2): 231-254.

Yi, B.-U. (2001). Compact Entailment and Wright's Verification Principle, Mind 110(438): 413-421.

Yi, B.-U. (2003). Wright and Suárez on the Verification Principle, Analysis 63(1): 58-61. 